\title{
Tobacco perceptions and practices: User groups and demographic characteristics, Mississippi, USA
}

Thomas J. Payne ${ }^{1,2}$, Joy L. Hart ${ }^{2,3}$, Aida L. Giachello ${ }^{2,4}$, Kandi L. Walker ${ }^{2,3}$, Wei Wang ${ }^{5}$, Allison Groom ${ }^{2,6}$, Mario Sims $^{2,7}$, Clara G. Sears $^{2,3}$, Alexander S. Lee ${ }^{2,3}$, Lindsay K. Tompkins ${ }^{2,3}$, Anshula Kesh ${ }^{2,8}$, Karen Robb ${ }^{2,6}$, Rose M. Robertson ${ }^{2,9}$

\author{
AFFILIATION \\ 1 Department of Otolaryngology-Head and Neck Surgery, University of \\ Mississippi Medical Center, Jackson, United States \\ 2 American Heart Association Tobacco Center for Regulatory Science, \\ Dallas, United States \\ 3 Department of Communication, University of Louisville, Louisville, \\ United States \\ 4 Department of Preventative Medicine, Northwestern University, \\ Chicago, United States \\ 5 U.S. Food and Drug Administration, Silver Spring, United States \\ 6 Customer and Marketing Research, American Heart Association, Dallas, \\ United States \\ 7 Department of Medicine, University of Mississippi Medical Center, \\ Jackson, United States
}

8 Office of Science Operations, American Heart Association, Dallas, United States 9 Science and Medicine Office, American Heart Association, Dallas, United States

\section{CORRESPONDENCE TO}

Joy L. Hart. Department of Communication, University of Louisville, Louisville, Kentucky, United States. E-mail: joy.hart@louisville.edu ORCID ID: https://orcid.org/0000-0003-3220-2638

\section{KEYWORDS}

tobacco, smoking, risk perception, age differences, racial differences

Received: 27 May 2020, Revised: 22 August 2020,

Accepted: 3 September 2020

https://doi.org/10.18332/popmed/127236 products as well as use and health information seeking behavior. The selection criteria and recruitment approach ensured a balance across race (black, white), age (18-34, >35 years), sex, and cigarette smoking status (current, former, never). Statistical analyses were performed using SAS (v.9.4). RESULTS Differences were observed across demographic subgroups regarding type and pattern of tobacco products used (e.g. mentholated, markers of nicotine dependence, hookah). Differences in preferred sources of health information based on age as well as perceptions of risk as a function of age, smoking status and race were also noted. Exposure to secondhand smoke and perceptions of its risks, quitting efforts and cessation methods differed by race.

CONCLUSIONS Study findings suggest key differences across important subgroups. Knowledge of such differences has the potential to improve strategic public health messaging, allowing health campaigns to more effectively prevent tobacco product uptake as well as promote interest in quitting tobacco.

\section{INTRODUCTION}

Since the release of the 1964 US Surgeon General's Report ${ }^{1}$, the prevalence of tobacco use in the United States has decreased substantially, although in recent years, the rate of reduction has slowed considerably ${ }^{2}$. Factors contributing to this diminished progress include more complex clinical presentation of smokers, greater dual and poly product use, and the upsurge in novel tobacco products ${ }^{3-5}$. Further, current public health messaging may be too generic to have an optimal motivational effect to further reduce the incidence of product uptake or to encourage current users to quit. Although information can be tailored to the interests, perceptions, and experiences of targeted audiences, often tobacco control efforts fail to adequately address such 
factors ${ }^{6}$. Because individuals are more likely to attend to information perceived as personally relevant ${ }^{7}$, tailored messaging for health campaigns seems especially useful.

A number of important differences have been identified across African American and Caucasian tobacco smokers in the United States. For example, African Americans generally begin smoking later than Caucasians, are far more likely to smoke mentholated products $^{8-10}$, smoke fewer cigarettes per day, and appear more nicotine dependent ${ }^{11-13}$. Less is known about other factors that differentiate these groups, such as attitudes and perceptions about specific tobacco products and relative health risks.

Further, the southeastern region of the United States continues to report some of the nation's highest tobacco use rates ${ }^{2}$. Thus, understanding tobacco-related views and behaviors in this region and developing targeted messaging strategies to reduce the health burden of tobacco-induced diseases is vital. This study examined tobacco-related perceptions and practices, with an emphasis on identifying differences across African American and Caucasian smokers. Toward this goal, we examined key demographic variables of race and age, and tobacco use characteristics.

\section{METHODS}

\section{Participants}

As part of a larger research program conducted by the American Heart Association Tobacco Center for Regulatory Science (TCORS), 284 participants from the Jackson, Mississippi metropolitan area were recruited to complete a questionnaire and take part in focus group interviews during 2014. For this exploratory study, a minimum of 200 participants was deemed necessary to identify response patterns in the qualitative (focus group) data, while providing an appropriate population number to detect large to medium sized effects in the survey data of somewhat unknown psychometric characteristics. Recruitment was conducted by a trained research assistant at various locations (e.g. clinics, shopping malls, community centers), each of which was visited at least 2-4 times per month over an 8-month period. To ensure our convenience sample included adequate representation of key study subgroups of the local population, recruitment efforts were geared toward filling predetermined cells based on gender, race, and age. Individuals agreeing to participate were scheduled for an appointment at a research clinic and received a $\$ 40$ stipend plus travel costs. This study was approved by the Institutional Review Board of the University of Mississippi Medical Center.

\section{Measures}

Participants completed screening and survey instruments designed to collect information across a variety of domains. The screening form contained 13 questions and required approximately five minutes to complete, and the questionnaire contained 64 questions and required 20-30 minutes to complete. This study focuses on responses to screening and survey items from the following topic areas: a) standard demographics, such as age, education, employment, income, and marital status; b) knowledge and perceptions general and tobacco product-specific health risks; sources, method, and level of exposure to health information resources; c) environmental tobacco smoke exposure; d) tobacco product use - type, amount, and duration; and e) quitting tobacco - attempts, success, and duration. Items were chosen or modified from existing surveys (e.g. PATH, PhenX) ${ }^{14,15}$ or investigator generated in those instances where items addressing desired topics were not available. All ratings by participants were based on 1-5 Likert scales.

For demographic and socioeconomic characteristics, participant responses were classified as follows: sex (male; female), race (Caucasian [CAUC]; African American [AA]), age (younger, 18-34 years; older, 35-64 years), education (high school or less; more than high school), marital status (married; single), and annual income $(<50000 ;>\$ 50000)$. In addition, participants were classified based on tobacco use status, utilizing criteria commonly employed in cigarette smoking studies: current smokers (SM) if they reported smoking 100 or more cigarettes in their lifetime and at least weekly current cigarette smoking; ex-smokers (EX) if they indicated no tobacco use of any type in the past 12 months but smoking 100 or more cigarettes in their lifetime; and never smokers (NS) if they indicated no tobacco use of any type in the past 12 months and between 1 and 99 cigarettes in their lifetime.

\section{Statistical analyses}

Primary analyses were conducted with models classified as: race by smoking status; and age by smoking status. Continuous variables were expressed as mean $\pm \mathrm{SD}$, and categorical and ordinal variables were expressed as frequency (\%). Continuous variables were compared with 2-way ANOVA, binary variables were compared with logistic regression models, and ordinal variables were compared with proportional odds models with race and smoking status as fixed effects. All primary analyses controlled for demographic variables (sex, education, marital status, income). Initial analyses revealed few significant interactions; thus, this report is based on main effects findings. Statistical analyses were performed using SAS (v.9.4) software (SAS Institute, Cary, NC) with statistical significance assessed at the 0.05 level.

\section{RESULTS}

Most participants were college graduates, especially EX and NS relative to $\mathrm{SM}(\mathrm{p}<0.001)$; most reported income as $<\$ 50000(\mathrm{p}<0.001)$; and most were single $(\mathrm{p}=0.002)$. Further, more women than men participated (females 175; males 109). Table 1 presents sample characteristics by race and smoking status.

Several differences were noted with respect to tobacco product use. Among SM, AA were far more likely than CAUC to use a mentholated product $(85.9 \%$ vs $22.6 \%$; $<<0.001)$ 
Table 1. Sample characteristics by racial group and smoking status

\begin{tabular}{|c|c|c|c|c|c|c|}
\hline & $\begin{array}{c}\text { CAUC SM } \\
(\mathrm{N}=31)\end{array}$ & $\begin{array}{l}\text { AA SM } \\
(N=80)\end{array}$ & $\begin{array}{c}\text { CAUC EX } \\
(\mathrm{N}=24)\end{array}$ & $\begin{array}{c}\text { AA EX } \\
(N=27)\end{array}$ & $\begin{array}{l}\text { CAUC NS } \\
(\mathrm{N}=21)\end{array}$ & $\begin{array}{c}\text { AA NS } \\
(\mathrm{N}=101)\end{array}$ \\
\hline Age (years) $($ Mean \pm SD) & $44.5 \pm 13.1$ & $42.4 \pm 13.6$ & $49.8 \pm 10.5$ & $47.4 \pm 12.6$ & $41.1 \pm 13.7$ & $40.9 \pm 14.0$ \\
\hline Married (\%) & 48.4 & 17.7 & 45.8 & 26.9 & 42.9 & 32.0 \\
\hline Education $(\%)<\mathrm{HS}$ & 53.3 & 55.9 & 25.0 & 44.4 & 23.9 & 18.9 \\
\hline Income $(\%)<\$ 50000$ & 91.3 & 95.6 & 77.3 & 86.4 & 55.0 & 80.9 \\
\hline
\end{tabular}

CAUC: Caucasian. AA: African American. SM: smoker. EX: ex-smoker. NS: never-smoker. HS: high school.

and age of smoking initiation was later in life (17.1 [SD=4.5] vs 13.2 [SD=3.3] years; $\mathrm{p}<0.001$ ). No difference was noted in time to first cigarette of the day. SM reported approximately 1.5 years longer total duration of use than EX (23.05 [12.2] vs 21.66 [12.6]; $p=0.022$ ), and CAUC used tobacco nearly 4.5 years longer than AA (25.51 [13.3] vs 21.12 [11.8]; $\mathrm{p}=0.016$ ). CAUC were more likely to have used hookah within the past month $(14.6 \%$ vs $5.3 \%$; $p=0.042)$. No difference was noted in likelihood of current vaping (past month), which averaged $4.1 \%$ across all groups.

With respect to health information, no differences were noted across race or smoking status for ratings of overall health risk knowledge, dangers associated with cigarettes, or subscriptions to email health-related services (endorsed by $24 \%$ of sample). Younger participants were more likely to indicate e-cigarettes/vaping was safer than cigarettes (2.76 [1.1] vs 3.08 [1.1]; $p=0.013$ ). Younger participants employed internet searches for health information more often than older participants (2.74 [1.2] vs 3.33 [1.2]; $p=0.004)$, as did SM relative to both EX and NS (3.61 [1.3] vs 3.00 [1.2]; $\mathrm{p}=0.033$ ), yet there were no differences in level of reported exposure to such information on the internet across any subgroups. Younger smokers reported greater likelihood of receiving health-related and tobacco information from school ( $23.6 \%$ vs $9.2 \%$; $\mathrm{p}=0.038$ ) and social media ( $30.3 \%$ vs $13.8 \%$; $\mathrm{p}=0.011$ ), whereas older smokers indicated employers (12.4\% vs $20.5 \%$; $\mathrm{p}=0.013)$ and newspapers/magazines (18.0\% vs $31.8 \%$; $\mathrm{p}=0.021$ ) were more frequent sources of such information.

A smoking status main effect for modality in searching for health information was significant $(\mathrm{p}=0.013)$, indicating SM were least likely to use desktops/laptops, whereas NS were most likely to employ these technologies $(35.1 \%$ vs $58.1 \%)$. No differences in smartphone use for this purpose were found. A race effect was also noted $(58.8 \%$ vs $42.5 \%$; $\mathrm{p}=0.013$ ) indicating CAUC used desktops/laptops more often than AA, but again no differences in smartphone use were found. Finally, an age main effect $(p<0.001)$ indicated older users were more likely to use desktops/laptops ( $56.4 \%$ vs $29.4 \%$ ), whereas younger users more often used smartphones ( $70.6 \%$ vs $26.2 \%)$.

CAUC reported that, in the past three months, they had greater exposure to tobacco health information than AA via internet access ( $50.0 \%$ vs $26.4 \%$; $<<0.001$ ), and SM reported less exposure overall relative to EX and NS (19.8\% vs $42.6 \%$ and $42.2 \%$, respectively; $\mathrm{p}<0.001$ ). SM also reported less health information exposure via social media $(13.2 \%$ vs $29.8 \%$ and $20.7 \%$, respectively; $\mathrm{p}=0.030$ ). Further, SM reported far less tobacco health information exposure via TV than other groups $(50.4 \%$ vs $61.7 \%$ and $65.5 \%$, respectively; $\mathrm{p}=0.007$ ), despite equivalent time spent watching TV across smoking status groups. AA indicated greater exposure to tobacco health information on billboards and signs placed on public transportation ( $22.6 \%$ vs $11.8 \%$; $\mathrm{p}=0.049$ ). Finally, although the overall level of concern about secondhand smoke was high across races, CAUC reported greater total exposure time (35.45 [44.52] vs 17.09 [24.70] hours/ week; $\mathrm{p}<0.001$ ) and believed secondhand smoke to be less dangerous relative to AA (1.6 [0.7] vs 1.4 [0.6]; $p=0.005$ ).

Examination of items related to quitting tobacco use revealed further differences. EX reported longer maximal quit duration relative to $\mathrm{SM}$, as expected $(\mathrm{p}=0.015)$, as did CAUC participants relative to AA ( $\mathrm{p}=0.023)$, and AA were less likely to have ever quit $(\mathrm{p}=0.012)$. CAUC participants were more likely to have tried bupropion $(20 \%$ vs $7.5 \%$; $\mathrm{p}=0.020)$ and varenicline $(43.6 \%$ vs $20.6 \% ; \mathrm{p}=0.038)$ to quit. No differences were noted in desire to quit, awareness of the tobacco quitline, or use of over-the-counter nicotine replacement products.

\section{DISCUSSION}

These findings suggest that race, smoking status and age are variously associated with tobacco product perceptions and practices. Overall, such findings have significant implications for improving the tailoring of tobacco control strategies to reduce health disparities. The span of such strategies is broad, ranging from advertising to cessation services. In particular, we believe the results of this study can facilitate the development of tailored messaging approaches to enhance the motivation of specific subgroups of smokers to seek tobacco-related health information and resources, eschew tobacco product consumption, and give greater consideration to quitting the use of tobacco products.

Differences in tobacco product use were consistent with findings in previous studies. For example, AA were more likely to smoke mentholated cigarettes and to begin 
smoking later in life $\mathrm{e}^{8-10}$; however, no differences across race were evident in time to first cigarette of the day, a marker for nicotine dependence ${ }^{9,12}$. No differences were noted in likelihood of having tried vaping; however, Caucasians were more likely to currently smoke hookah.

With respect to health knowledge, several findings are noteworthy. First, younger users rated vaping as safer than cigarettes, consistent with previous research ${ }^{13,16}$. Although the health-related consequences of vaping (e.g. potential gateway to other nicotine products, lower rates of quitting smoking among vapers, differential risk across health conditions) have been a highly contentious topic within the tobacco control community ${ }^{17-20}$, the perspectives held by youth are important considerations that require more in-depth study (e.g. to clarify the accuracy of specific beliefs and how these relate to product use). Second, the locations and manner in which individuals receive health-related information differed by subgroups: younger participants received more information from school, social media, and use of smartphones; for older persons, the sources reported most often were employers, newspapers/magazines and internet searches using desktops/laptops. Such findings suggest important strategies for choosing media modalities when presenting targeted messages. Related findings with similar implications include that Caucasian participants had greater exposure to tobaccorelated health information on the internet, whereas current smokers reported less overall exposure to such information, specifically with respect to social media and TV. Given that there were no differences in time spent watching TV across groups, this finding seems likely due to program and website choices or selective attention/recall. Finally, our finding that African Americans indicated greater exposure to health information in the outdoor environment, such as buses and billboards, may be due to factors including the locations of these signs and/or greater use of public transportation by this group; however, additional research is required to more fully examine reasons for this finding. It seems likely that certain media options represent better choices for reaching specific subgroups of individuals.

Differences in quitting behavior were observed as well. In particular, a pattern of longer term quitting among Caucasian participants was observed, along with a greater likelihood of using the prescription medications varenicline and bupropion. Encouraging African American smokers to speak with physicians about using available prescription medications would seem to be an important implication, as use of these treatment options is associated with higher quit rates ${ }^{21,22}$.

Taken together, these findings indicate significant differences across identified subgroups regarding tobacco use characteristics, perceptions and attitudes about tobacco use, the manner in which health information is sought, and methods employed to achieve cessation. Differences in perceptions and behavior suggest the potential value of targeted messaging that addresses those issues, as well as identifying opportunities to provide corrective information and recommend options not being fully employed. Delivering information that is currently lacking in messaging approaches for particular subgroups may increase resolve not to try tobacco in non-smokers as well as motivation to quit, and the use of more effective intervention strategies for smokers. We recommend greater efforts to conduct systematic research to test these assumptions and explore those issues most likely to increase impact.

\section{Limitations}

There are some limitations to this study that should be acknowledged. The sample is based on African American and Caucasian individuals residing in central Mississippi; thus, generalizations to other locations and other races/ethnicities should be made with caution. Given that recruitment goals for balancing race, age, gender and smoking status were achieved, the impact of this concern is somewhat minimized. Although the sample size $(n=284)$ was adequate to yield meaningful differences across key subgroups, replication is warranted. The data are based on self-reports, which raises the possibility of associated biases (e.g. recall); however, concordance with previous research suggests these findings are accurate and meaningful.

\section{CONCLUSIONS}

This examination of perceptions and practices based on demographics and tobacco use status reveals important information that differentiates subgroups of individuals in a manner that is of value for comprehensive tobacco control. First, expected differences, primarily across race, were observed. Some expected differences in tobacco product usage were noted, as well as new findings related to nicotine dependence, vaping, and hookah. We observed substantial differences in health beliefs, knowledge and information seeking as a function of age and race. Finally, important differences in quitting behavior/methods were reported by race. These findings have the potential to contribute to the development of tailored messaging that can help members of vulnerable populations to better appreciate the risks associated with tobacco use, refrain from experimenting with tobacco or consider quitting, and use effective strategies to achieve abstinence.

\section{REFERENCES}

1. U. S. Department of Health, Education, and Welfare. Smoking and health: Report of the Advisory Committee to the Surgeon General of the Public Health Service. In: Public Health Service Publications, No. 1103. https://www.govinfo. gov/content/pkg/GPO-SMOKINGANDHEALTH/pdf/GPOSMOKINGANDHEALTH.pdf. Published 1964. Accessed August 22, 2020.

2. Creamer MR, Wang TW, Babb S, et al. Tobacco product use and cessation indicators among adults-United States, 2018. MMWR Morb Mortal Wkly Rep. 2019;68(45):1013-1019. doi:10.15585/mmwr.mm6845a2

3. Rostron BL, Schroeder MJ, Ambrose BK. Dependence 
symptoms and cessation intentions among US adult daily cigarette, cigar, and e-cigarette users, 2012-2013. BMC Public Health. 2016;16(1):814. doi:10.1186/s12889-016-3510-2

4. Wang TW, Gentzke AS, Creamer MR, et al. Tobacco product use and associated factors among middle and high school students-United States, 2019. MMWR Surveill Summ. 2019;68(12):1-22. doi:10.15585/mmwr.ss6812a1

5. Wang TW, Asman K, Gentzke AS, et al. Tobacco product use among adults-United States, 2017. MMWR Morb Mortal Wkly Rep. 2018;67(44):1225-1232. doi:10.15585/mmwrmm6744a2

6. World Health Organization. WHO report on the global tobacco epidemic, 2011: Warning about the dangers of tobacco. https://apps.who.int/iris/bitstream/ handle/10665/44616/9789240687813_eng.pdf;jsessionid= 1FCC8C57C5413D57F4FFF6A007519497? sequence $=1$. Published 2011. Accessed August 22, 2020.

7. Petty RE, Cacioppo JT. Involvement and persuasion: Tradition vs. integration. Psych Bull. 1990;107(3):367-374. doi:10.1037/0033-2909.107.3.367

8. Giovino G, Villanti AC, Mowery PD, Sevilimendu V, Niaura $\mathrm{R}$, Vallone DM, Abrams DB. Differential trends in cigarette smoking in the USA: Is menthol slowing progress? Tob Control. 2015;24(1):28-37. doi:10.1136/tobaccocontrol-2013-051159

9. Payne T], Diefenbach L. Characteristics of African-American smokers: A brief review. Am J Med Sci. 2003;326(4):212-215. doi:10.1097/00000441-200310000-00012

10. Smith PH, Assefa B, Kainth S, Salas-Ramirez KY, McKee SA, Giovino GA. Use of mentholated cigarettes and likelihood of smoking cessation in the United States: A meta-analysis. Nicotine Tob Res. 2020;22(3):307-316. doi:10.1093/ntr/ntz067

11. Benowitz NL, Dains KM, Dempsey D, Wilson M, Jacob P. Racial difference in the relationship between number of cigarettes smoked and nicotine and carcinogen exposure. Nicotine Tob Res. 2011;13(9):772-783. doi:10.1093/ntr/ntr072

12. Perez-Stable EJ, Herrera B, Jacob P, Benewitz NL. Nicotine metabolism and intake in black and white smokers. JAMA, 1998;280(2):152-156. doi:10.1001/jama.280.2.152

13. Weinberger AH, Platt JM, Smith PH, Goodwin RD. Racial/ ethnic differences in self-reported withdrawal symptoms and quitting smoking 3 years later: A prospective, longitudinal examination of US adults. Nicotine Tob Res. 2017;19(3):373-378. doi:10.1093/ntr/ntw221

\section{ACKNOWLEDGEMENTS}

We extend special thanks to Elandra Felix who conducted participant recruitment and various study activities, and Debbra Hunter who assisted with administrative aspects of study implementation.

\section{CONFLICTS OF INTEREST}

The authors have each completed and submitted an ICMJE form for disclosure of potential conflicts of interest. The authors declare that they have no competing interests, financial or otherwise, related to the current work. All authors report grants from National Institutes of Health during the conduct of the study.

\section{FUNDING}

This work was supported by the National Heart, Lung, and Blood
14. Population Assessment of Tobacco and Health (PATH). https://pathstudyinfo.nih.gov/UI/HomeMobile.aspx. Published 2018. Accessed August 22, 2020.

15. PhenX Consensus Measures for Phenotypes and Exposures. Phenx Home. https://www.phenx.org/. Accessed August 22, 2020.

16. Vu TT, Groom A, Hart JL, et al. Socioeconomic and demographic status and perceived health risks of e-cigarette product contents among youth: Results from a national survey. Health Promot Pract. 2020;21(1_suppl):148S-156S. doi:10.1177/1524839919882700

17. Choi K, Foster JL. Beliefs and experimentation with electronic cigarettes: A prospective analysis among young adults. Am J Prev Med. 2014;46(2):175-178. doi:10.1016/j.amepre.2013.10.007

18. McMillen RC, Gottlieb MA, Shaefer RM, Winickoff JP, Klein JD. Trends in electronic cigarette use among US adults: Use is increasing in both smokers and non-smokers. Nicotine Tob Res. 2015;17(10):1195-1202. doi:10.1093/ntr/ntu213

19. Prochaska JJ, Grana RA. E-cigarette use among smokers with serious mental illness. PLoS One. 2014;9(11):e113013. doi:10.1371/journal.pone.0113013

20. U. S. Department of Health and Human Services. E-cigarette use among youth and young adults: A report of the Surgeon General-executive summary. Atlanta, GA: U.S. Department of Health and Human Services, Centers for Disease Control and Prevention, National Center for Chronic Disease Prevention and Health Promotion, Office on Smoking and Health; 2016. https://e-cigarettes.surgeongeneral.gov/documents/2016_ SGR_Exec_Summ_508.pdf. Accessed August 22, 2020.

21. World Health Organization. WHO Framework Convention on Tobacco Control: Electronic nicotine delivery systems and electronic non-nicotine delivery systems (ENDS/ENNDS): Report by WHO. https://www.who.int/fctc/cop/cop7/FCTC_ COP_7_11_EN.pdf?ua=1. Published August 2016. Accessed August 22, 2020.

22. Anthenelli RM, Benowitz NL, Wet R, et al. Neuropsychiatric safety and efficacy of varenicline, bupropion, and nicotine patch in smokers with and without psychiatric disorders (EAGLES): A double-blind, randomized, placebo-controlled clinical trial. Lancet. 2016;387(10037):2507-2520. doi:10.1016/S0140-6736(16)30272-0

Institute (NHLBI) of the National Institutes of Health (NIH) and FDA Center for Tobacco Products under Award Numbers P50HL120163 and U54HL120163. The content is solely the responsibility of the authors and does not necessarily represent the official views of the NIH, the Food and Drug Administration, or the American Heart Association. The funding sponsors had no role in study design; data collection, analyses, or interpretation; manuscript preparation; or the decision to publish the results.

\section{PROVENANCE AND PEER REVIEW}

Not commissioned; externally peer reviewed. 